\title{
Spectroscopic Studies of Metal Binding and Metal Selectivity in Bacillus Subtilis BSco, a Homolog of the Yeast Mitochondrial Protein Sco1p
}

\author{
Supplementary Material
}

Luisa Andruzzi§, Michiko Nakano§, Mark J. Nilges†, and Ninian J. Blackburn§*

$\S$ Department of Environmental and Biomolecular Systems, OGI School of Science and Engineering, OHSU, Beaverton, OR 97006-8921

$\dagger$ Illinois EPR Research Center, University of Illinois at Urbana-Champaign, 600 South Mathews Ave, Urbana, Illinois 61801 


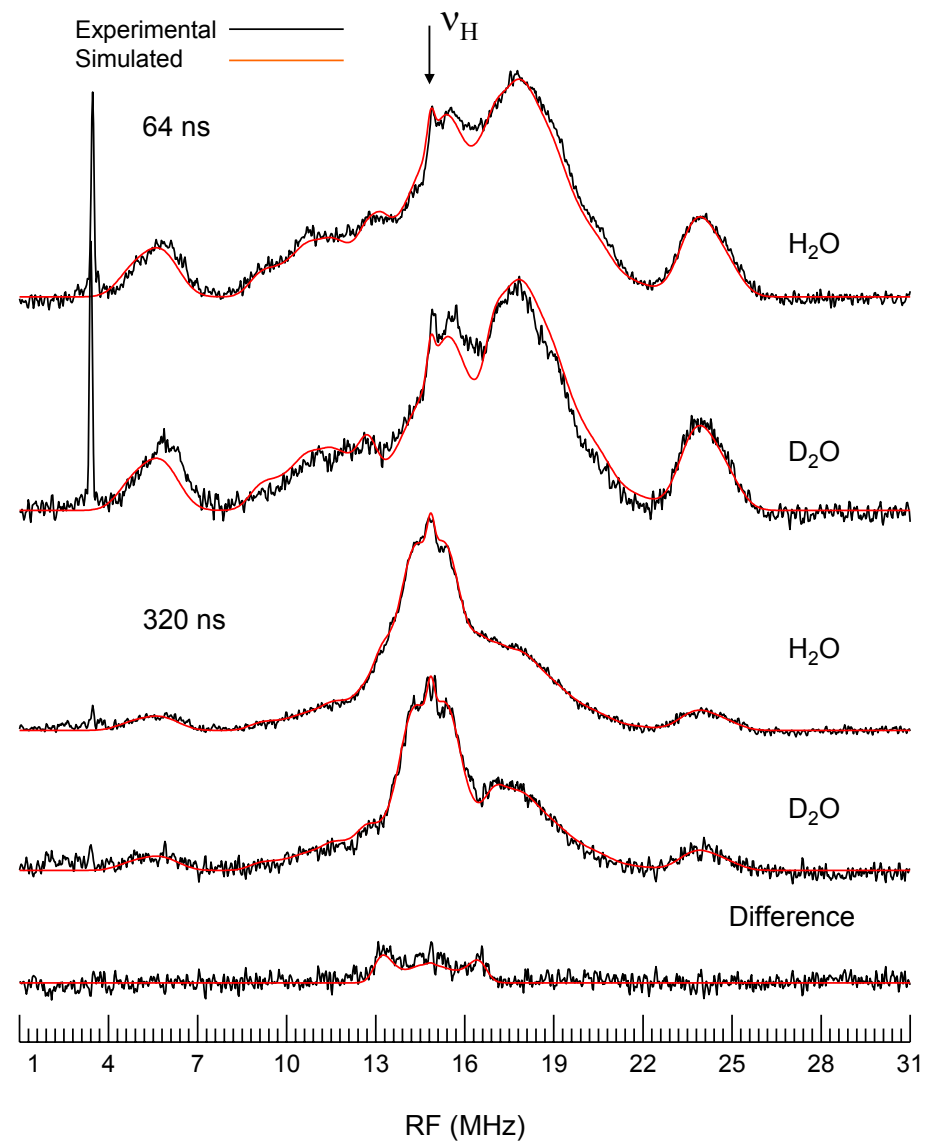

Figure S1. Experimental (dark) and simulated (light) X-band (9.67 GHz) ENDOR spectra of $\mathrm{Cu}(\mathrm{II}) \mathrm{BSco}$ in $\mathrm{H}_{2} \mathrm{O}$ and $\mathrm{D}_{2} \mathrm{O}$ for $\mathrm{t}_{\pi}$ equal to 64 and 320 ns. The magnetic field was set at 3489 Gauss. 

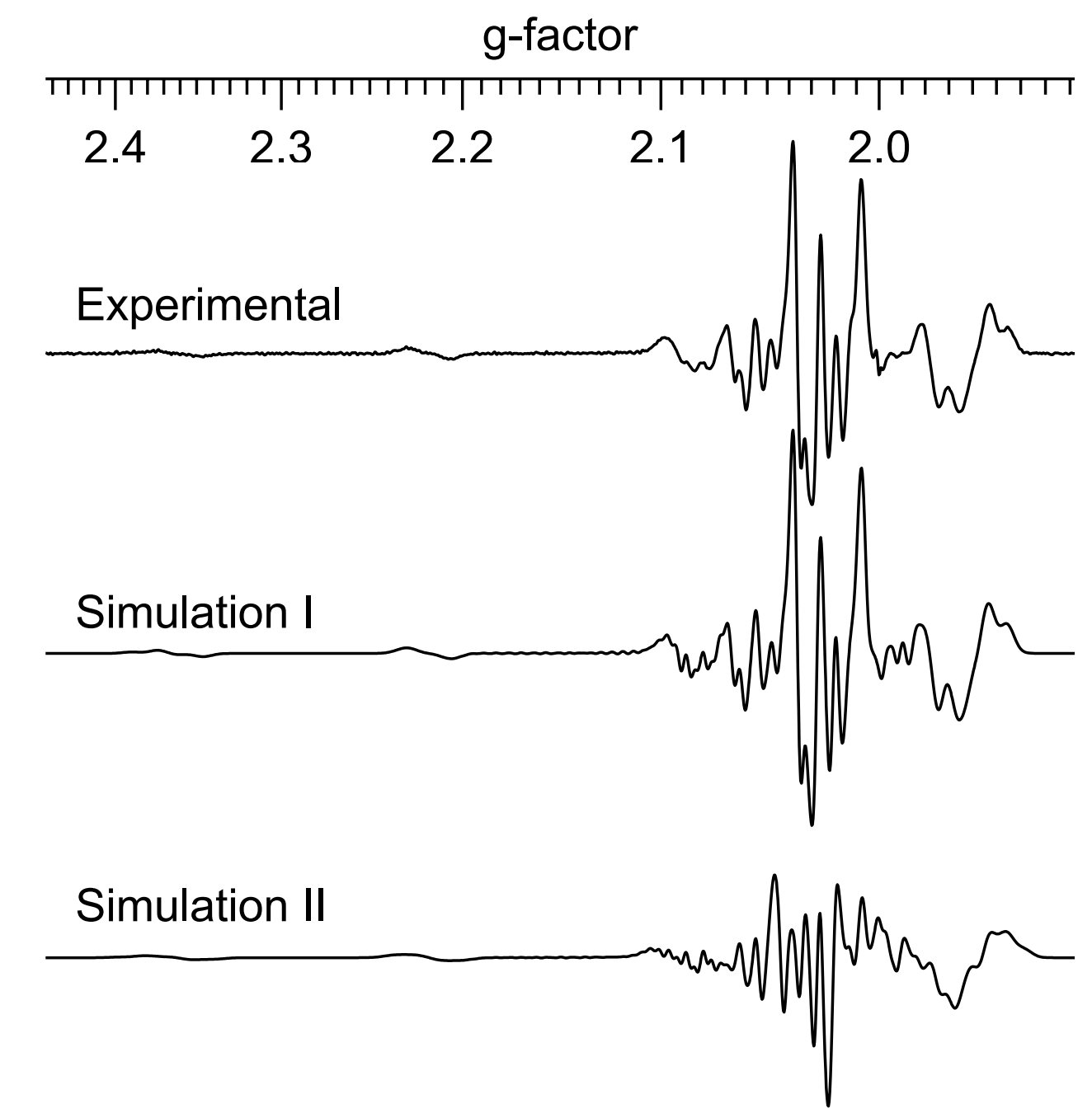

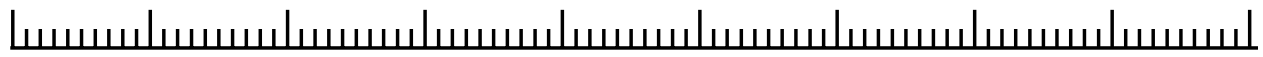
2648272828082888296830483128320832883368
MAGNETIC FIELD (GAUSS)

Figure S2. Experimental and simulated second derivative X-band $(9.055 \mathrm{GHz})$ EPR spectra of $\mathrm{Cu}(\mathrm{II}) \mathrm{BSco}$ in $\mathrm{D}_{2} \mathrm{O}$. Simulation I is the best-fit using one nitrogen and one proton. Simulation II shows the effect of using two equivalent nitrogen's and one proton with the same parameters as in Simulation I. Optimization of Simulation II did not give significant improvement in the fit. In particular, the use of two equivalent nitrogens causes the superhyperfine splitting envelope to become too wide to properly fit the experimental spectrum. 


\section{The Program SIMPIPM}

Program SIMPIPM includes strain-broadening effects calculated using exact gradients of the spin-Hamiltonian matrix. The program assumes that the strains in the system can be attributed to a Gaussian distribution of the spin-Hamiltonian parameters and that, under the assumption that the variances in the spin Hamiltonian parameters are small, the strain will give rise to Gaussian line broadening. ENDOR spectra were simulated using SIMEND, which is a version of SIMPIP that calculates a frequencyswept spectrum and includes an intensity weighting function for the Boltzman population and for angle-selection. The angle selection is:

$$
I_{u v}=\sum_{(C u) m I} \sum_{(N) m I}\left[\begin{array}{l}
\sum_{n} T M_{u n}\left(\beta_{u}-\beta_{n}\right) F\left(\left(H_{r e s}-H_{u n}\left({ }^{C u} m_{I},{ }^{N} m_{I}, \theta, \varphi\right), \Delta H(\vartheta, \varphi)+\right.\right. \\
\sum_{m} T M_{m v}\left(\beta_{m}-\beta_{v}\right) F\left(H_{r e s}-H_{m v}\left({ }^{C u} m_{I},{ }^{N} m_{I}, \theta, \varphi\right), \Delta H(\vartheta, \varphi)\right)
\end{array}\right]
$$

where the copper and nitrogen splittings of $\mathrm{H}_{\mathrm{un}}$ and $\mathrm{H}_{\mathrm{mv}}$ are calculated using firstorder perturbation theory, and the anisotropic Gaussian linewidth $\Delta \mathrm{H}(\theta, \varphi)$ is obtained by fitting the residual linewidth due to the remaining nuclei (in the case of the nitrogen ENDOR, the (N) $m_{I}$ contribution is dropped) . The contributions from the individual protons and the nitrogens are calculated separately. Because the sensitivity decreases with frequency due to instrumentation, an empirical weighting or roll-off function was added:

$$
G(v)=\frac{1}{1+\left(v / v_{0}\right)^{n}}
$$


where $\mathrm{n}$ is found to be around 2.9 and $v_{0}$ is approximately $3.5 \mathrm{MHz}$. This correction is an empirical one that also includes corrections due to other sources such as relaxation.

\section{Extended Discussion of parameters obtained by simulation of EPR spectra.}

The linewidth was modeled assuming strain in $\mathrm{g}$ and A (Table 3). While the gstrain was greatest along the z-direction as expected, there was minimal strain in A along z. For most copper complexes, the correlated strain in $\mathrm{g}$ and A gives rise to clearly visible $\mathrm{m}_{\mathrm{I}}$ dependent linewidths for the four parallel ${ }^{63,65} \mathrm{Cu}$ hyperfine lines ${ }^{1,2}$. Here the linewidths of the four parallel lines were found to be independent of $\mathrm{m}_{\mathrm{I}}$. Simulation of the EPR spectra did show some strain for $\mathrm{A}_{\perp}$, in particular for the y-direction.

The $\mathrm{g}$ and ${ }^{63,65} \mathrm{Cu}$ hyperfine tensors are roughly collinear with the noncoincidence between the z-axes being only 1 degree. Because the nitrogen and proton hyperfine tensors are nearly axial, only two Euler angles can be determined to any accuracy; the third Euler angle, $\gamma$, is set to $0^{\circ}$ or $-90^{\circ}$ depending on whether the rotation by $\beta$ is about the $\mathrm{y}$ - or x-axis, respectively. For the nitrogen hyperfine tensor the direction of the major hyperfine component corresponds roughly to the $\mathrm{g}_{\mathrm{y}}$ axis. The proton hyperfine tensor shows the least coincidence with $\mathrm{g}$.

Simulations gave a copper nuclear quadrupole coupling of QD $=4.85 \mathrm{MHz}$. This value is slightly less than that seen in type 1 complexes $\left(5-6 \mathrm{MHz}^{3}\right)$ but slightly greater than that seen for $\mathrm{S}_{2} \mathrm{O}_{2}$ complexes $\left(3.9 \mathrm{MHz}{ }^{4}\right)$. The nuclear quadrupole coupling has a large rhombic component $(\eta>1)$.

\section{Analysis of Nitrogen quadrupole ESEEM peaks}

The peaks at $0.73,1.6$, and $4.0 \mathrm{MHz}$ are characteristic of the remote nitrogen of a copper-coordinated imidazole ${ }^{5}$. The first two peaks are narrow and intense and are characteristic of a weakly coupled nitrogen for which the hyperfine coupling is mainly isotropic and nearly cancels twice the nuclear Zeeman energy. These peaks represent the pure quadrupole frequencies ${ }^{6}$ : 


$$
v_{0}=\frac{1}{2} e^{2} q Q \eta \quad v_{-}=\frac{1}{4} e^{2} q Q(3-\eta) \quad v_{+}=\frac{1}{4} e^{2} q Q(3+\eta)
$$

If $\eta \approx 1$, the $v_{0}$ and $v_{\text {-peaks }}$ will be degenerate and as such correspond to the peak at 0.73 , while the peak at 1.6 corresponds to $v_{+}$and is consistent with $\left|e^{2} q Q\right|(4 / 3$ QD) equal to about 1.6 MHz. The third and broader peak at 4.0 MHz is due to one of two $\Delta \mathrm{M}_{\mathrm{I}}=2$ transition or double-quantum transitions whose frequencies are given by:

$$
v_{d q}=2\left[\left(v_{n} \pm a / 2\right)^{2}+\left(e^{2} q Q / 4\right)^{2}\left(3+\eta^{2}\right)\right]^{1 / 2}
$$

The 4.0 MHz peak is consistent with a hyperfine coupling around $1.5 \mathrm{MHz}$, which is typical of copper-bound imidazoles ${ }^{6}$.

\section{Analysis and Interpretation of $\beta$-methylene splittings.}

The two cysteines are expected to give rise to four approximately equal $\beta$ methylene splittings. Analysis of the ENDOR spectra gives only three protons, H1, H2, and $\mathrm{H} 3$, as candidates for the four $\beta$-methylene protons. Proton hyperfine couplings for the histidine are expected to be no larger than $5 \mathrm{MHz}^{8}$, while $\beta$-methylene proton splittings for type 1 copper complexes range from 16 to $31 \mathrm{MHz}^{7}$. The assignment of $\mathrm{H} 2$ $\left(\mathrm{a}_{\text {iso }}=8.4\right)$ and, in particular, $\mathrm{H} 1\left(\mathrm{a}_{\mathrm{iso}}=18.5\right)$ to a cysteine ligand is quite likely.

Simulation of the EPR spectrum showed that the strong coupling for $\mathrm{H} 1$ is due to only a single proton, suggesting the assignment of $\mathrm{H} 2$ to the second $\beta$-methylene proton, although the assignment of $\mathrm{H} 2$ to the second cysteine ligand cannot be ruled out. The large $\beta$-methylene splittings observed for copper type 1 complexes have been attributed to delocalization of spin density into a sulfur $\mathrm{p} \pi$ orbital. The resulting hyperfine coupling on the two $\beta$-methylene protons is due to hyperconjugation, which gives rise to a coupling which is dependant on the dihedral angle between the p $\pi$ orbital on the sulfur and the $\mathrm{C}_{\beta}-\mathrm{H}_{\beta}$ bond ${ }^{9}$.

$$
A^{H 1}=\rho_{s}\left[B \cos ^{2} \theta+C\right] \quad A^{H 2}=\rho_{s}\left[B \cos ^{2}\left(\theta+120^{\circ}\right)+C\right]
$$

where $\mathrm{B} \sim 100 \mathrm{MHz}$ and $\rho_{\mathrm{s}}$ is the $\mathrm{p} \pi$ spin density. The $\mathrm{C}$ term is usually small and can usually be neglected for $\pi$-systems. From the ratio of the hyperfine splittings for $\mathrm{H} 1$ and $\mathrm{H} 2$ and from the above equation we estimate dihedral angles of $\theta=11^{\circ}$ and $\theta+120^{\circ}=$ $131^{\circ}$ and a $\mathrm{p} \pi$ spin density of $\approx 0.19$. Assuming the $\mathrm{p} \pi$ orbital lies perpendicular to the 
$\mathrm{Cu}-\mathrm{S}-\mathrm{C}_{\beta}$ plane as it does in type 1 complexes, the corresponding complementary $\mathrm{Cu}-\mathrm{S}$ $\mathrm{C}_{\beta}-\mathrm{H}_{\beta}$ dihedral angles will be $79^{\circ}$ and $-41^{\circ}$. For blue copper type 1 complexes, the ratio of hyperfine splittings for $\mathrm{H} 1$ and $\mathrm{H} 2$ is much closer to one, and the $\mathrm{Cu}-\mathrm{S}-\mathrm{C}_{\beta}-\mathrm{H}_{\beta}$ dihedral angles for the two $\beta$-methylene protons are closer to $\pm 60^{\circ}$. In Werst, D. E.; Davoust, C. E.; Hoffman, B. M. J. Am. Chem. Soc. 1991, 113, 1533-1538, the Cu-S-C ${ }^{\beta}-\mathrm{H}^{\beta}$ dihedral angle and dihedral angle between the $\mathrm{p} \pi$ orbital on the sulfur and the $\mathrm{C}^{\beta}-\mathrm{H}^{\beta}$ bond are both referred to as theta and appear to have been equated, the net result is that the deviation of the $\mathrm{Cu}-\mathrm{S}-\mathrm{C}^{\beta}-\mathrm{H}^{\beta}$ dihedral angles from $\pm 60^{\circ}$ should be much larger than reported and the value of C is much too large (see Veselov, A.; Olesen, K.; Sienkiewicz, A.; Shapleigh, J. P.; Scholes, C. P. Biochemistry 1998, 37, 6095-6105, and footnote 6 therein). However, for the case of the blue-green copper type 1 complex, nitrite reductase, quite inequivalent values have been reported for the two $\beta$-methylene protons ${ }^{10}$. In particular, the reported values of 20 and $8.7 \mathrm{MHz}$ for $\mathrm{H} 1$ and $\mathrm{H} 2$ are quite similar to our values of 18.5 and 8.5 $\mathrm{MHz}$. This can be rationalized in light of the fact that nitrite reductase has a CT spectra which is indicative of a complex with both type 1 (tetrahedral) and type 2 (tetragonal) character. Optimal formation of a $\mathrm{Cu}$-cys $\pi$-bond requires the $\mathrm{Cu}-\mathrm{S}$ bond to lie between the lobes of the copper $d_{x 2-y 2}$ orbital and for the $S \mathrm{p} \pi$ orbital to lie in the $x y$ plane. Thus, the inequivalence in $\mathrm{H} 1$ and $\mathrm{H} 2$ may arise from a less than optimal orientation of the cysteine with respect to the $\mathrm{Cu} \mathrm{d}_{\mathrm{x} 2-\mathrm{y} 2}$ orbital and with the resultant weakening of the $\mathrm{p} \pi$ bond.

The $\mathrm{H} 3$ proton could be assigned to a second cysteine, but with a value of $\mathrm{a}_{\text {iso }}$ of $5.1 \mathrm{MHz}$ it could also be assigned to a proton on the imidazole ring (values of 4.6 and 1.5 $\mathrm{MHz}$ are predicted by scaling data for $\mathrm{Cu}$ (imidazole $){ }_{4}^{8}$ by the value of the near ${ }^{14} \mathrm{~N}$ coupling). Even if the $\mathrm{H} 3$ proton could be assigned to a second cysteine, its relatively small value implies that $\mathrm{p} \pi$ bonding is weak and delocalization of the unpaired spin is relatively small. Once more, for square planar geometry, a strong $\mathrm{p} \pi$ bond is not expected. In general, the formation of $\mathrm{Cu}-\mathrm{S} \mathrm{p} \pi$ bond is the exception rather than a rule ${ }^{11}$. For a strict square planar complex a $\mathrm{Cu}-\mathrm{S} \mathrm{p} \pi$ bond should not be able to form, since the $\mathrm{Cu}-\mathrm{S}$ bond would lie along the lobe of the $\mathrm{d}_{\mathrm{x} 2 \mathrm{y} 2}$ orbital. In this case, the $\beta$-methylene couplings are expected to be anisotropic and relatively small. The second cysteine then may be coordinated in a more optimal orientation for square planar geometry giving rise 
to a strong $\mathrm{Cu}-\mathrm{S}$ p $\sigma$ bond. The data are thus consistent with the $\mathrm{Cu}-\mathrm{N}$ and one $\mathrm{Cu}-\mathrm{S}$ bond lying close to the lobes of the $\mathrm{d}_{\mathrm{x} 2-\mathrm{y} 2}$ orbital while the other $\mathrm{Cu}-\mathrm{S}$ bond lying off axis, somewhere between the lobe of the $d_{x 2-y 2}$ orbital and the bisector between the lobes of the $\mathrm{d}_{\mathrm{x} 2-\mathrm{y} 2}$ orbital.

\section{Analysis and Interpretation of Exchangeable proton splittings.}

For square-planar geometry a fourth ligand is expected, mostly likely an O ligand since nitrogen coupling is observed for only a single nitrogen ligand. $\mathrm{D}_{2} \mathrm{O}$ experiments have revealed the presence of one exchangeable proton, presumably from an $\mathrm{OH}^{-} / \mathrm{H}_{2} \mathrm{O}$. Assuming a point-dipole mode with the spin density localized on the copper atom: $A_{\text {dip }}=\left(\mu_{0} / 4 \pi\right) g \beta g_{n} \beta_{n}\left(3 \cos ^{2} \theta-1\right) / h r^{3}$

the $\mathrm{Cu}-\mathrm{H}$ distance can be estimated as $3.7 \AA$ with the $\mathrm{Cu}-\mathrm{O}$ distance being up to $1 \AA$ shorter. For equatorial $\mathrm{H}_{2} \mathrm{O}$ coordination, a $\mathrm{Cu}-\mathrm{O}$ distance of $\sim 2 \AA$ and principal hyperfine couplings of 3,7 , and $10 \mathrm{MHz}$ are expected ${ }^{12}$. As such, this weakly coupled proton is more likely due to weakly coordinated $\mathrm{OH}^{-} / \mathrm{H}_{2} \mathrm{O}$ possibly having an axial ligation. As the difference in EPR linewidths between $\mathrm{D}_{2} \mathrm{O}$ and $\mathrm{H}_{2} \mathrm{O}$ media would allow a much larger proton coupling and as the largest component of the A-strain is in the xy plane, one cannot rule the possibility that there exists a $\mathrm{OH}-/ \mathrm{H}_{2} \mathrm{O}$ molecule with larger hyperfine couplings but because of strain its ENDOR spectrum is broaden so that it is not observed.

\section{References to the extended discussion}

(1) Antholine, W. E.; Hanna, P. M.; McMillin, D. Biophys. J. 1993, 64, 267-272.

(2) Yuan, H.; Perille Collins, M. L.; Antholine, W. E. Biophys. J. 1999, 76, 2223 2229.

(3) Roberts, J. E.; Cline, J. F.; Lum, V.; Freeman, H.; Gray, H. B.; Peisach, J.; Reinhammar, B.; Hoffman, B. M. J. Am. Chem. Soc. 1984, 106, 5324-5330.

(4) White, L. K.; Belford, R. L. J. Am. Chem. Soc. 1976, 98, 4428-4438.

(5) Mims, W. B.; Peisach, J. J. Chem. Phys. 1978, 69, 4291-4930. 
(6) Jiang, F., McCracken, J., and Peisach, J., J. Am. Chem. Soc., 1990, 112, 90359044.

(7) Werst, D. E.; Davoust, C. E.; Hoffman, B. M. J. Am. Chem. Soc. 1991, 113, 1533-1538.

(8) Van Camp, H. L.; Sands, R. H.; Fee, J. A. J. Chem. Phys. 1981, 75, 20982107.

(9) Gordy, W. Theory and applications of electron spin resonance; Wiley: New York, 1980.

(10) Veselov, A.; Olesen, K.; Sienkiewicz, A.; Shapleigh, J. P.; Scholes, C. P. Biochemistry 1998, 37, 6095-6105.

(11) Daugherty, R. G.; Wasowicz, T.; Gibney, B. R.; DeRose, V. J. Inorg. Chem. 2002, 41, 2623-2632.

(12) Atherton, N. M.; Horsewill, A. J. Mol. Phys. 1979, 37, 1349-1361. 